\title{
Choosing a stable housekeeping gene and protein is essential in generating valid gene and protein expression results
}

\author{
M Sullivan-Gunn', E Hinch', V Vaughan' and P Lewandowski*, I \\ 'Molecular Nutrition Unit, School of Medicine, Deakin University, Waurn Ponds 3217, Australia
}

British Journal of Cancer (201 I) 104, 1055. doi:10.1038/bjc.20II.35 www.bjcancer.com

Published online I March 20I I

(c) 20II Cancer Research UK

Sir,

We have read and support the concerns of Caradec et al (2010) in the British Journal of Cancer regarding the stability of GAPDH as an internal control gene that was used in the recent study by Kontos et al (2010) to normalise L-DOPA decarboxylase mRNA as a prognostic marker in colorectal adenocarcinoma. We too have recently found variance in protein and gene expression of the commonly used housekeeping genes, GAPDH and ribosomal $18 \mathrm{~s}$, in the hearts of colon adenocarcinoma-bearing mice. Although differences in GAPDH mRNA were not statistically significant, results show some variation in GAPDH protein levels between murine adenocarcinoma (MAC) models of tumour-bearing cachectic (MAC16) and non-cachectic mice (MAC13; $P=0.05$ ). We have also found significant variation in the gene expression of ribosomal 18s in the hearts of these two MAC cancer models $(P<0.001)$ and in each cancer model compared with non-tumourbearing control mice $(P=0.004 ; P<0.001)$. Further to this, variations in ribosomal $18 \mathrm{~s}$ expression were observed at different stages of cancer development in the hearts of MAC13 tumour-bearing mice compared with control mice. Ribosomal 18s gene expression in the hearts of MAC13 tumourbearing mice showed significant differences at days 12

\section{REFERENCES}

Caradec J, Sirab N, Revaud D, Keumeugni C, Loric S (2010) Is GAPDH a relevant housekeeping gene for normalisation in colorectal cancer experiments? Br J Cancer 103: $1475-1476$

De Santis C, Smith-Keune C, Jerry D (2010) Normalizing RT-qPCR data: are we getting the right answers? An appraisal of normalization approaches and internal reference genes from a case study in the Finfish Lates calcarifer. Mar Biotechnol (NY) (e-pub ahead of print 23 March 2010; doi:10.1007/s10126-010-9277-z)

Kontos C, Papadopoulos I, Fragoulis E, Scorilas A (2010) Quantitative expression analysis and prognostic significance of L-DOPA decarboxylase in colorectal adenocarcinoma. Br J Cancer 102: 1384-1390

Lundby C, Nordsborg N, Kusuhara K, Kristensen K, Neufer P, Pilegaard H (2005) Gene expression in human skeletal muscle: alternative
$(P=0.011)$ and $21(P=0.047)$, when compared with day 29 after implantation.

Although still widely used as housekeeping genes, GAPDH and ribosomal 18s expression levels have been shown to vary under different experimental conditions (Mahoney et al, 2004; De Santis et al, 2010; Nelissen et al, 2010), and disease states (Mahoney et al, 2004; De Santis et al, 2010; Nelissen et al, 2010) showed GAPDH to be highly variable in cancerous compared with non-cancerous tissue. Nelissen et al (2010) recently described GAPDH and ribosomal $18 \mathrm{~s}$ as the most unstable housekeeping genes under different experimental conditions in qPCR experiments with rat oligodendrocytes. The use of variable housekeeping genes as normalisers will lead to misinterpretation and irrelevant results.

Thoughtful consideration as well as evaluation of a valid housekeeping gene that is ubiquitously expressed and stable across sample groups is absolutely necessary to obtain reliable gene expression results and eliminate misinterpretations. Alternatively, fluorescein-labeled oligonucleotides such as OliGreen (Invitrogen, Mulgrave, Victoria, Australia), which measures cDNA content in each sample, may be a more reliable reference for normalising protein and gene expression results (Lundby et al, 2005; Rhinn et al, 2008). normalization method and effect of repeated biopsies. Eur J Appl Physiol 95: $351-360$

Mahoney D, Carey K, Fu M, Snow R, Cameron-Smith D, Parise G, Tarnopolsky M (2004) Real-time RT-PCR analysis of housekeeping genes in human skeletal muscle following acute exercise. Physiol Genomics 18: $226-231$

Nelissen K, Smeets K, Mulder M, Hendriks J, Ameloot M (2010) Selection of reference genes for gene expression studies in rat oligodendrocytes using quantitative real time PCR. J Neurosci Methods 187: 78-83

Rhinn H, Scherman D, Escriou V (2008) One-step quantification of single-stranded DNA in the presence of RNA using Oligreen in a real-time polymerase chain reaction thermocycler. Anal Biochem 372: $116-118$ 